\title{
Adenovirus-mediated ING4/PTEN double tumor suppressor gene co-transfer modified by RGD enhances antitumor activity in human nasopharyngeal carcinoma cells
}

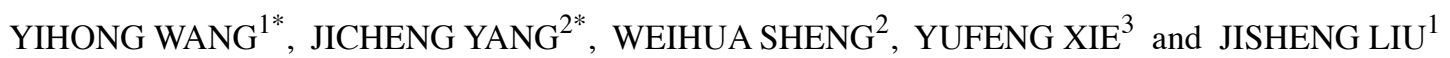 \\ ${ }^{1}$ Department of ENT, The First Affiliated Hospital of Soochow University; ${ }^{2}$ Cell and Molecular Biology Institute, \\ College of Medicine, Soochow University; ${ }^{3}$ Institute for Cancer Center Laboratory, The First Affiliated Hospital \\ and Experiment Center of Soochow University, Suzhou 215006, P.R. China
}

Received October 27, 2014; Accepted December 17, 2014

DOI: $10.3892 / \mathrm{ijo} .2015 .2822$

\begin{abstract}
Inhibitor of growth-4 (ING4) is a member of the inhibitor of growth (ING) family and acts as a tumor suppressor protein. PTEN is a phosphatase and shows potent and extensive antitumor activity. In this study, we constructed an RGD-modified bicistronic ING4/PTEN adenovirus (Ad.RGD-ING4-PTEN) and comprehensively investigated its effects following modification of the CNE human nasopharyngeal carcinoma cell line both in vitro and in vivo. We demonstrated that Ad.RGD-ING4-PTEN enhanced growth inhibition and apoptosis. Furthermore, expression of P21, Bax and cleaved caspase-3 was upregulated, while that of Bcl-2 and survivin was downregulated in CNE cells and CNE xenografted tumors. Moreover, Ad.RGD-ING4-PTEN treatment additively downregulated CD34, VEGF and microvessel density in subcutaneously (s.c.) xenografted CNE cell tumors. The enhanced antitumor activity generated by Ad.RGDING4-PTEN was closely associated with activation of the intrinsic and extrinsic apoptotic pathways and additive inhibition of tumor angiogenesis both in vitro and in vivo. On the basis of this evidence, it is believed that cancer gene therapy combining two tumor suppressors such as ING4 and PTEN can be used to establish an effective and novel therapeutic strategy for nasopharyngeal carcinoma and other cancers.
\end{abstract}

\section{Introduction}

Nasopharyngeal carcinoma (NPC), which is a type of malignant tumor that originates from the epithelium of the nasopharynx,

Correspondence to: Dr Jisheng Liu, Department of ENT, The First Affiliated Hospital of Soochow University, Suzhou 215006, P.R. China

E-mail: ljswwq@sina.com

*Contributed equally

Key words: ING4,PTEN, RGD, nasopharyngeal carcinoma, enhanced antitumor effect is relatively common in China, South-East Asia, North Africa, Alaska and parts of the Mediterranean basin. During the progression of NPC, distant metastasis and early cervical lymph node metastasis may occur, representing a serious clinical problem $(1,2)$. The main treatments for NPC are radiotherapy and adjuvant chemotherapy; however, the total 5-year survival rate is $<40 \%$ and these treatments are associated with a series of side-effects and complications. Consequently, patient tolerance and compliance is not good. Therefore, effective and safe novel therapeutics for NPC are urgently required (3-5).

Cancer gene therapy is a new strategy that shows great potential for the treatment of tumors. This approach depends on the introduction of hereditary material into cells to generate a biological effect. Recombinant adenovirus expression vectors are frequently used for this purpose. NPC is characterized by a multistep process of molecular and genetic changes in oncogenes and tumor suppressor genes. Therefore, the efficacy of single gene-mediated NPC therapy is limited and a multigenebased combination approach may be more effective.

RGD peptides are a class of short peptides containing arginine-glycine-aspartic acid (Arg-Gly-Asp), which function mainly in the role of cell adhesion to fibronectin. Extracellular matrix proteins and adhesion proteins in the blood containing RGD sequences, together with the integrins which serve as their receptors, constitute a major recognition system for cell adhesion. RGD sequences have high affinity for $\alpha v \beta 3$ integrins, which are usually expressed at high levels in tumor cells and vascular endothelial cells in tumors (6); consequently, RGD containing proteins have been used as carriers to deliver drugs or genes into tumor cells (7). Some studies have used RGD-modified polymers or liposome as non-viral vectors for delivering genetic material to improve the efficiency of cancer gene therapy, including anti-angiogenic therapy $(8,9)$. A class of RGD peptides called RGD-4C has been shown to bind specifically to $\alpha v \beta 3$ integrins by phage display technology. In this study, the capsid protein encoded by the adenoviral vectors was transformed to express RGD-4C to facilitate targeted adherence to tumor cells and improve the efficiency of infection.

Inhibitor of growth 4 (ING4), a member of the ING tumor suppressor family that was first discovered by Shiseki et al (10), is attracting increasing attention as novel candidate 
tumor suppressor gene. ING4, which is the best characterized member of the ING protein family (11) has been shown to interact with different structures, including histone modification complexes such as histone deacetylase (HDAC) and histone acetyltransferase (HAT). Structural and biochemical analyses have shown that ING4 is composed of a flexible nuclear localization sequence (NLS), an N-terminal domain, and a plant homeodomain (PHD) finger that is formed from a homodimer with obvious affinity for H3K4me3. The $\mathrm{N}$-terminal domain enables the formation of homodimers by permitting independent folding, which leads to the formation of a coiled structure (12). Several studies have demonstrated that ING4 expression is commonly decreased or lost at the RNA and protein levels in human tumors such as in HNSCC, hepatocellular carcinoma, melanoma, ovarian and brain cancers (13-16). ING4 has been shown to inhibit tumor cell growth and to induce cell apoptosis in different tumor types such as hepatocellular, lung and pancreatic carcinomas (17-19). Furthermore, non-physiological overexpression of ING4 inhibits cell proliferation and induces $\mathrm{G} 2 / \mathrm{M}$ cell cycle arrest (20). ING4 can also inhibit the activity $N F-\kappa B$ and HIF-1 $\alpha$, and interact with liprin $\alpha 1$ to suppress tumor angiogenesis, invasion and metastasis.

Phosphatase and tensin homolog deleted on chromosome ten (PTEN) is another tumor suppressor, the activity of which is lost through various mechanisms in many diverse forms of cancer (21-23). PTEN is a lipid phosphatase and dual-specificity protein that blocks phosphatidylinositol 3 kinase (PI3K) signaling by converting phosphatidylinositol-(3,4,5)-triphosphate (PIP3) into phosphatidylinositol $(4,5)$-bisphosphate (PIP2). This negatively regulates PIP3-dependent processes such as the activity of protein kinase B (AKT) and phosphatidylinositol-dependent kinase-1 (PDK1) to inhibit cell growth and metabolism, cell cycle progression and migration. Accumulating evidence shows that PTEN has significant PIP3independent functions. PTEN protein phosphatase activity is important for the inhibition of cellular migration mediated by PTEN (24). Furthermore, several studies have confirmed that PTEN is able to exit and exist outside the cell $(25,26)$.

These observations implicate ING4 and PTEN as promising tumor suppressors that can negatively modulate tumor growth via diverse pathways. On the basis of the antitumor characteristics of ING4 and PTEN, we speculated that combination therapy comprising ING4 and PTEN would lead to an intensive antitumor effect; however, the therapeutic potential of a combination of ING4 and PTEN for NPC has not yet been reported. Therefore, in this study, we constructed an RGD-modified bicistronic ING4 and PTEN recombinant adenoviral vector (Ad.RGD-ING4-PTEN). We analyzed its combined therapeutic effect on human NPC cells in vitro and in vivo in an athymic nude mouse xenografted tumor model and also explored its potential molecular mechanisms.

\section{Materials and methods}

All animals received humane care according to the guidelines of the Guidebook for the Care and Use of Laboratory Animals (16). The study protocol was approved by the Animal Research Ethics Committee at the First Affiliated Hospital of Soochow University (Suzhou, China).
Adenoviruses, cell lines, reagents and mice. The Ad.RGDING4-PTEN and Ad.RGD-green fluorescent protein (GFP) replication-incompetent Ad5E1- and E3-deleted adenoviruses and the QBI-293A human embryonic kidney cell line were kindly provided by Dr Jicheng Yang (Cell and Molecular Biology Institute, College of Medicine, Soochow University, Suzhou, China). The human NPC CNE cell line was purchased from the American Type Culture Collection (Shanghai, China). The CNE and QBI-293A cells were cultured in RPMI-1640 (Gibco, Shanghai, China) supplemented with $10 \%$ fetal bovine serum (Hyclone, Shanghai, China). The reverse transcriptase MuMLV and TRIzol were purchased from Invitrogen (Shanghai, China).

The following reagents were used in this study: 3-(4,5-dimethylthiazol-2-yl)-2,5-diphenyltetrazolium bromide (MTT) kit (Sigma, Shanghai, China); Annexin V-PE/7-AAD apoptosis detection kit and propidium iodide (PI) staining kit (BD Biosciences, Shanghai, China); antibodies specific for ING4, P21, Bax, Bcl-2, VEGF, CD34 and $\beta$-actin (Santa Cruz, Shanghai, China); antibodies specific for PTEN, survivin and caspase-3 (Cell Signaling, Shanghai, China); PageRuler Prestained Protein Ladder (Fermentas, Shanghai, China); Dylight 800-Labeled Antibody (KPL, Shanghai, China); UltraSensitive ${ }^{\text {TM }}$ SP kit (Maixin, Fuzhou, China); SuperEnhanced chemiluminescence detection kit (Applygen Technologies, Beijing, China).

Male BALB/c athymic nude mice (aged 4 weeks) were purchased from Shanghai Experimental Animal Center (Shanghai, China) and were raised in a specific pathogen-free environment in the Laboratory Animal Center of Soochow University according to the Regulations for the Administration of Affairs Concerning Experimental Animals. All experimental protocols were approved by the Institutional Animal Care and Use Committee.

Analysis of the adenoviral infection efficiency with and without $R G D$ modification. The infection efficiency of adenoviruses with and without RGD modification was investigated to assess the optimal multiplicity of infection (MOI) for maximal adenoviral infection and transgene expression in CNE tumor cells. CNE NPC cells were infected with Ad-GFP, Ad-ING4, Ad-PTEN, Ad-ING4-PTEN, Ad.RGD-GFP, Ad.RGD-ING4, Ad.RGD-PTEN or Ad.RGD-ING4-PTEN at various MOIs $(0,1,10,25,50,100$ and 200). After $48 \mathrm{~h}$, the infection efficiency was assessed according to GFP expression observed by fluorescence microscopy. Following infection of CNE cells with Ad-GFP, Ad-ING4, Ad-PTEN, Ad-ING4-PTEN, Ad.RGD-GFP, Ad.RGD-ING4, Ad.RGD-PTEN or Ad.RGDING4-PTEN (MOI, 50), GFP expression was analyzed by flow cytometry.

ING4/PTEN transgene expression in CNE tumor cells. The adenovirus-mediated transcriptional expression of ING4 and PTEN in CNE cells was determined by RT-PCR and western blot analysis. Total RNA was extracted from Ad.RGD-GFP-, Ad.RGD-ING4-, Ad.RGD-PTEN- or Ad.RGD-ING4PTEN-infected and uninfected CNE cells using TRIzol. First-strand cDNA was reverse-transcribed using RNA as the template and oligo d(T)18 as the primer. RT-PCR was carried out using cDNA as the template and ING4-F (5'-GCGT CGACATGGATGATGGGATGTATTTGGAAC-3'), ING4-R (5'-GCAAGCTTCTATTTCTTCTTCCGTTCTTGGGAG-3'), 
PTEN-F (5'-GCGGTACCATGACAGCCATCATCAAA GAG-3'), PTEN-R (5'-CGAAGCTTTCAGACTTTTGTAAT TTGTGT-3'), GAPDH-F (5'-TGATGACATCAAGAAGGTG GTGAA-3') and GAPDH-R (5'-TCCTTGGAGGCCATGTGG GCC-3') as primers. All of the RT-PCR products were analyzed by $2 \%$ agarose gel electrophoresis. Total cellular lysates derived from Ad.RGD-GFP, Ad.RGD-ING4, Ad.RGD-PTEN and Ad.RGD-ING4-PTEN-infected and uninfected CNE cells were resolved by $12 \%$ sodium dodecyl sulfate-polyacrylamide gel electrophoresis (SDS-PAGE), then transferred onto a polyvinylidene fluoride membrane. The membrane was blocked with 5\% skimmed milk solution and then the incubated with the appropriate primary antibody [mouse anti-ING4 $(1: 1,000)$, anti-PTEN $(1: 1,000), \beta$-actin $(1: 1,000)]$. The membrane was washed with TBST three times and incubated with Dylight 800-Labeled Antibody. Finally, the membrane was photographed on a fluorescence imager. The experiment was repeated three times.

Cell viability assay. The in vitro cytotoxic effect of Ad.RGDING4-PTEN on the CNE cells was assessed by MTT assay. The CNE cells were dispensed into 96-well culture plates at $1 \times 10^{4}$ cells per well and then treated with (Ad.RGD, Ad.RGD-ING4, Ad.RGD-PTEN or Ad.RGD-ING4-PTEN) or without the adenovirus (PBS control) at the optimal MOI of 50 for the indicated time periods ( $0-4$ days) after 24 -h incubation at $37^{\circ} \mathrm{C}$. The viability of CNE cells was evaluated using an MTT kit according to the manufacturer's protocol before treatment and at different four points after treatment.

Flow cytometric analysis of the cell cycle and apoptosis. Cell cycle analysis of CNE cells was performed by flow cytometry following PI staining. CNE cells $\left(1 \times 10^{5}\right)$ were cultured with (Ad.RGD-ING4-PTEN, Ad.RGD-ING4, Ad.RGD-PTEN or Ad.RGD) or without adenovirus (PBS control) at the optimal MOI of 50. After $72 \mathrm{~h}$, cells were collected, washed twice in cold PBS and fixed in $70 \%$ cold alcohol $\left(>12 \mathrm{~h}\right.$ at $\left.4^{\circ} \mathrm{C}\right)$. Cells were then washed in cold PBS again and stained with PI solution at $37^{\circ} \mathrm{C}$ for $30 \mathrm{~min}$ in the dark. Apoptosis was evaluated by Annexin V-PE/7-AAD double staining following the manufacturer's instructions. In brief, adenovirus-treated cells were collected, washed in cold PBS and incubated with $5 \mu \mathrm{l}$ Annexin V-PE, $5 \mu 1$ 7-AAD and $100 \mu 11 \mathrm{X}$ binding buffer for $15 \mathrm{~min}$ at room temperature in the dark. A further $400-\mu 1$ $1 \mathrm{X}$ binding buffer was then added to the cells and apoptosis was analyzed by flow cytometry.

Western blot analysis. CNE cells $\left(1 \times 10^{7}\right)$ were infected with (Ad.RGD-ING4-PTEN, Ad.RGD-ING4, Ad.RGD-PTEN or Ad.RGD) or without adenovirus (PBS control) at the optimal MOI of 50. After $48 \mathrm{~h}$, the cells were collected, washed with cold PBS and lysed in $1 \mathrm{ml}$ lysis buffer. Total cellular proteins were isolated and the protein concentration was determined spectrophotometrically in BCA protein assays. To explore the molecular mechanism involved in Ad.RGD-ING4-PTEN enhancement of growth inhibition and apoptosis and changes in the cell cycle, CNE cell lysates (100 $\mu \mathrm{g}$ per lane) were subjected to western blot analysis as described previously using primary antibodies specific for survivin, caspase-3, Bcl-2, Bax and P21.
Animal studies. Male athymic nude mice were hypodermically injected on the right anterior axilla with $5 \times 10^{6} \mathrm{CNE}$ cells and then monitored daily for tumor growth. Tumor dimensions were measured with calipers and the volume was calculated according to the following formula: tumor size $=a b^{2} / 2$, where $\mathrm{a}$ and $\mathrm{b}$ are the larger and smaller dimensions, respectively. When the tumor reached $\sim 200 \mathrm{~mm}^{3}$, CNE cell xenografted tumor-bearing mice ( $\mathrm{n}=5$ per group) were intratumorally injected with PBS (PBS control) or $1 \times 10^{9}$ pfu of Ad.RGDING4-PTEN, Ad.RGD-ING4, Ad.RGD-PTEN and Ad.RGD (a total of 6 doses on alternate days). Tumor volume was then measured every other day and tumor-bearing mice were sacrificed by cervical dislocation 20 days after treatment. All of the xenografted tumors were weighed, fixed with $10 \%$ neutral formalin and embedded in paraffin for hematoxylin and eosin (H\&E) staining and immunohistochemical analysis.

Immunohistochemical analysis. CD34, VEGF, survivin, caspase-3, Bcl-2, Bax and P21 expression by treated and untreated CNE s.c. xenografted tumors was investigated by immunohistochemistry using the UltraSensitive ${ }^{\mathrm{TM}}$ SP kit according to the manufacturer's instructions. Microvessel density (MVD) was determined by CD34 immunostaining as previously described by Weidner (27). Any endothelial cell cluster that was immunoreactive for CD34 and was clearly separated from adjacent microvessels was defined as a single countable vessel. The mean number of microvessels or integral optical density (IOD) of immunohistochemical intensity counted in five randomly selected fields of view under high-power microscopy (x200) was calculated by Image-Pro Plus 6.0 software (Media Cybernetics, Bethesda, MD, USA).

Evaluation of synergistic interaction. The interactive effects of ING4 and PTEN in CNE cells following RGD-modified adenovirus-mediated ING4 and PTEN coexpression were evaluated in terms of the Q-value calculated according to the formula (28), $\mathrm{Q}=\mathrm{F}(\mathrm{A}+\mathrm{B}) / \mathrm{FA}+(1-\mathrm{FA}) \mathrm{FB}$, where $\mathrm{F}(\mathrm{A}+\mathrm{B})$ represents the fraction of cells affected by Ad.RGD-ING4PTEN treatment compared with the untreated control group, FA represents the fraction of cells affected by Ad.RGD-ING4 alone, and FB represents the fraction of cells affected by Ad.RGD-PTEN alone. A value of $\mathrm{Q}>1.15$ indicates a synergistic effect between ING4 and PTEN, Q $<0.85$ indicates an antagonistic effect and $\mathrm{Q}$ between 0.85 and 1.15 indicates an additive effect.

Statistical analysis. All data are presented as the mean values $\pm \mathrm{SD}$. The significance of the difference between groups was evaluated by one-way and two-way repeated measures analysis of variance (ANOVA) and multiple comparisons with SPSS 10.0 software. A value of $\mathrm{P}<0.05$ was considered statistically significant.

\section{Results}

Infection efficiency of adenoviruses with and without RGD modification. More than $95 \%$ of CNE tumor cells transfected with Ad.RGD-GFP, Ad.RGD-ING4, Ad.RGD-PTEN, and Ad.RGD-ING4-PTEN at 50 MOI or Ad-GFP, Ad-ING4, Ad-PTEN, and Ad-ING4-PTEN at 100 MOI showed 

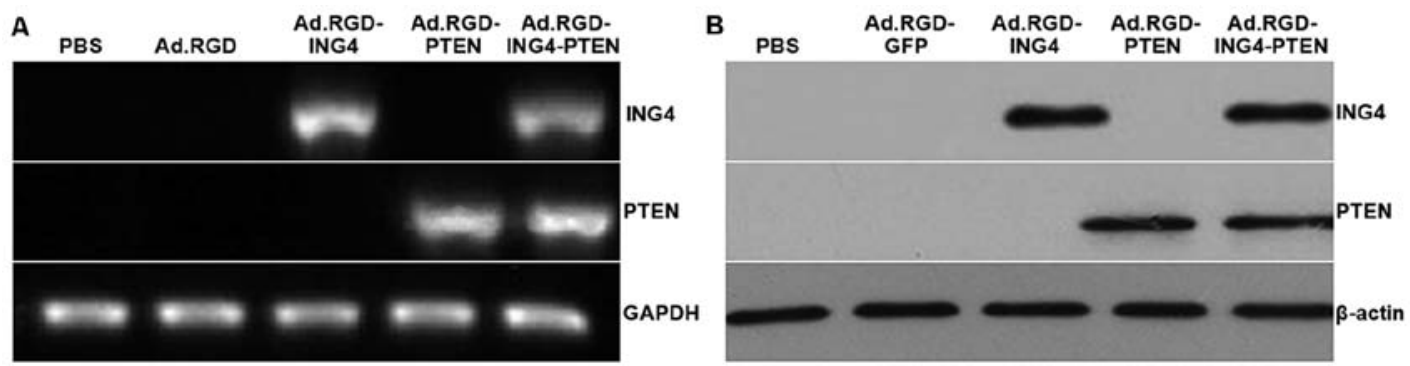

Figure 1. Adenovirus-mediated ING4 and PTEN coexpression in CNE human nasopharyngeal carcinoma cells. (A) RT-PCR analysis of adenovirus-mediated ING4 and PTEN transcriptional expression. Total cellular RNAs were obtained from Ad.RGD-ING4-PTEN-, Ad.RGD-ING4-, Ad.RGD-PTEN- and Ad.RGDinfected and uninfected CNE tumor cells. First-strand cDNAs were synthesized from RNA using reverse transcriptase; PCRs were conducted using primer sets specific for ING4, PTEN, and the housekeep gene GAPDH. (B) Western blot analysis of adenovirus-mediated ING4 and PTEN translational expression. Total cellular lysates derived from Ad.RGD-ING4-PTEN-, Ad.RGD-ING4-, Ad.RGD-PTEN- and Ad.RGD-infected and uninfected CNE tumor cells were analyzed by immunoblotting with anti-ING4, anti-PTEN and anti- $\beta$-actin. Data shown are representative of three independent experiments.
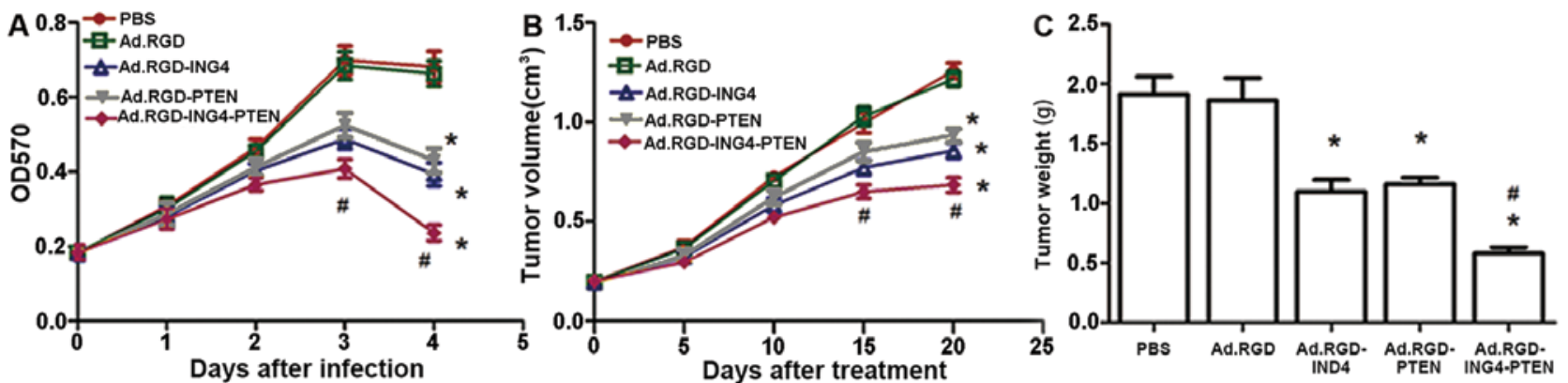

Figure 2. Ad.RGD-ING4-PTEN induced enhanced tumor suppression in CNE human nasopharyngeal carcinoma cells. (A) The cytotoxic effect of Ad.RGDING4-PTEN on CNE human nasopharyngeal carcinoma cells in vitro. CNE cells were treated with Ad.RGD-ING4-PTEN, Ad.RGD-ING4, Ad.RGD-PTEN or Ad.RGD (blank adenovirus control) at the optimal MOI of 50 (PBS served as a control) for the indicated time periods (0-4 days). Cell survival was assessed at days $0,1,2,3$ and 4 by MTT assay. ${ }^{*} \mathrm{P}<0.05$ vs. PBS and Ad.RGD groups; ${ }^{*} \mathrm{P}<0.05$ vs. Ad.RGD-ING4 and Ad.RGD-PTEN groups (Q=1.01 and 1.06 at days 3 and 4 after treatment, respectively); one-way repeated measures ANOVA and multiple comparisons, $\mathrm{n}=3$ replicates per condition. (B and C) Ad.RGD-ING4-PTEN enhanced the antitumor effect on xenografted CNE tumors in vivo. Athymic nude mice bearing subcutaneously xenografted tumors were intratumorally injected with Ad.RGD-ING4-PTEN, Ad.RGD-ING4, Ad.RGD-PTEN, Ad.RGD or PBS (a total of 6 doses on alternate days). The CNE xenografted tumor volume (B) was measured before and after treatment and xenografted tumors were isolated at 20 days after treatment and tumor weight (C) was measured. ${ }^{*} \mathrm{P}<0.05$ vs. PBS and Ad.RGD groups; ${ }^{*} \mathrm{P}<0.05$ vs. Ad.RGD-ING4 and Ad.RGD-PTEN groups $\left(\mathrm{Q}_{\mathrm{volume}}=0.98\right.$ and 1.02 at days 15 and 20 after treatment, respectively, and $\mathrm{Q}_{\text {weight }}=1.07$ ), one-way and two-way repeated measures ANOVA and multiple comparisons ( $\mathrm{n}=5$ mice per condition). Data shown are representative of three independent experiments.

GFP expression, with no abnormalities in the cell form observed. Flow cytometry showed that the infection rate of RGD-modified adenoviruses at 50 MOI was $>96 \%$ compared with $75 \%$ for unmodified adenoviruses. Therefore, we selected 50 MOI RGD-modified adenoviruses as an optimal dose for transfection of CNE human NPC cells in this study.

Ad.RGD-ING4-PTEN transgene expression. RT-PCR and western blot analysis of infected and uninfected CNE cells revealed transgene expression of both ING4 and PTEN at the transcriptional and translational levels in the Ad.RGDING4-PTEN-infected CNE cells (Fig. 1). ING4 and PTEN expression was also found in Ad.RGD-ING4- and Ad.RGDPTEN-infected CNE cells, respectively, but neither ING4 nor PTEN expression was detected in Ad.RGD-GFP-infected or uninfected CNE cells.

Increased tumor suppression by ING4 and PTEN coexpression . To investigate whether combined ING4 and PTEN treatment led to an enhanced antitumor effect, we coexpressed the ING4 and PTEN tumor suppressor genes by RGD-modified adeno- virus-mediated co-transfer and evaluated its combined effect on CNE human NPC cells. The CNE tumor cells were infected with Ad.RGD-ING4-PTEN, Ad.RGD-ING4, Ad.RGD-PTEN or Ad.RGD at the optimal MOI of 50. The viability of CNE tumor cells infected in vitro with Ad.RGD-ING4-PTEN was tested daily before and for 4 days after treatment using MTT assays. As shown in Fig. 2A, compared with the Ad.RGD and PBS control groups, adenovirus-mediated ING4 and/or PTEN expression obviously suppressed CNE cell growth in vitro in a time-dependent manner with peak inhibition at day 4 after infection $(\mathrm{P}<0.05)$. Moreover, combined ING4 and PTEN coexpression induced additive antitumor activity against $\mathrm{CNE}$ tumor cells compared with the Ad.RGD-ING4- and Ad.RGDPTEN-treated groups $(\mathrm{P}<0.05 ; \mathrm{Q}=1.01$ and 1.06 at days 3 and 4 after treatment, respectively). To further investigate whether the combination of ING4 with PTEN enhanced antitumor efficacy in vivo, athymic nude mice ( $\mathrm{n}=5$ per group) bearing s.c. xenografted CNE tumors were intratumorally injected with Ad.RGD-ING4-PTEN, Ad.RGD-ING4, Ad.RGD-PTEN or Ad.RGD (100 $\mu 1,1 \times 10^{9} \mathrm{pfu} / 1$ on alternate days). The tumor volume (Fig. 2B) was recorded on alternate days. Xenografted 


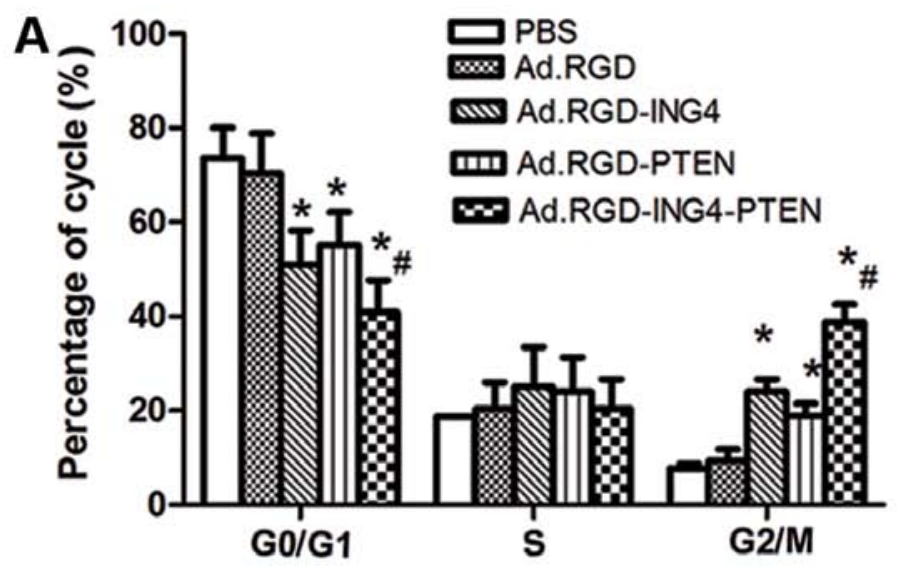

C
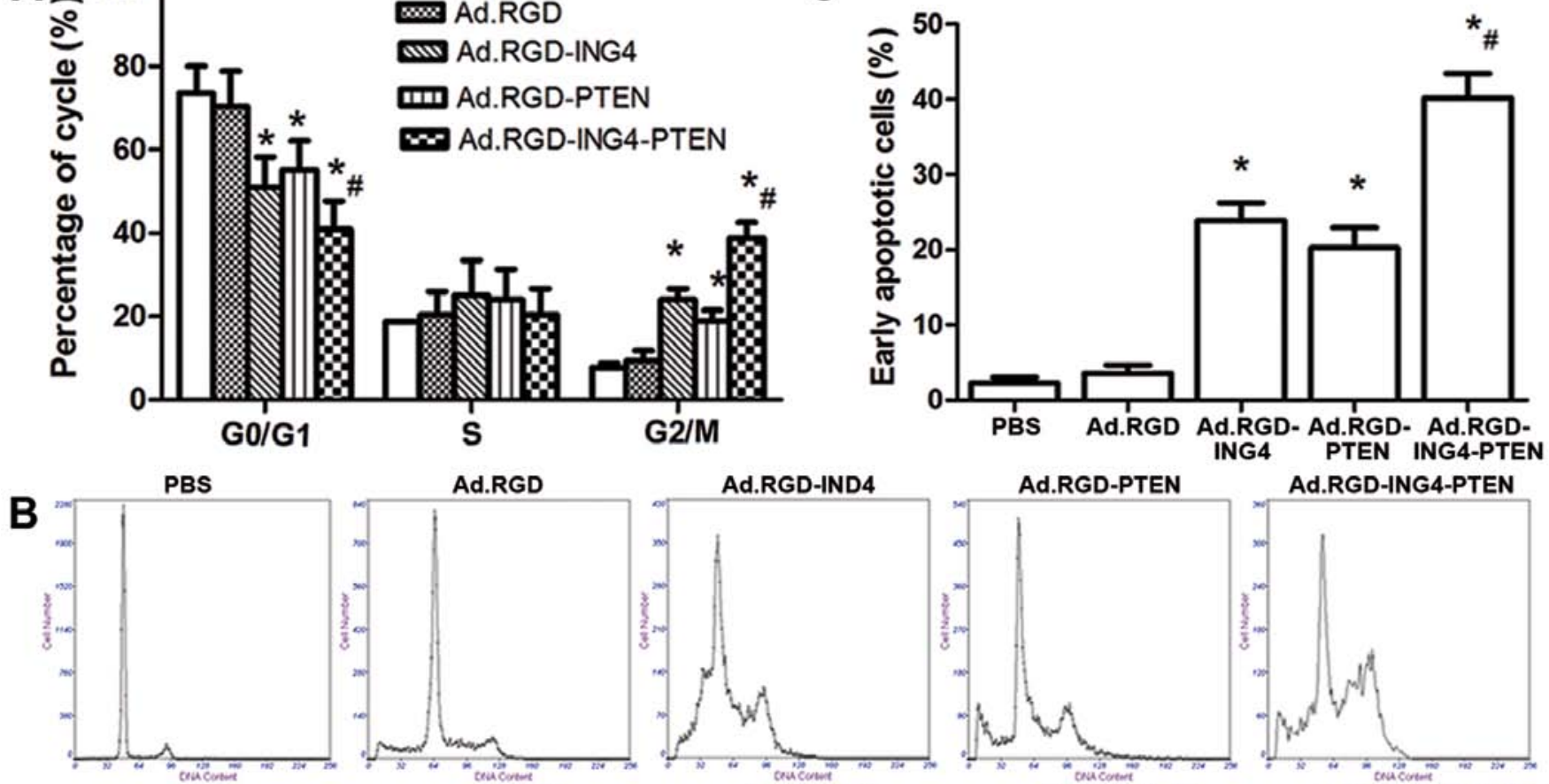

Ad.RGD

Ad.RGD-IND4
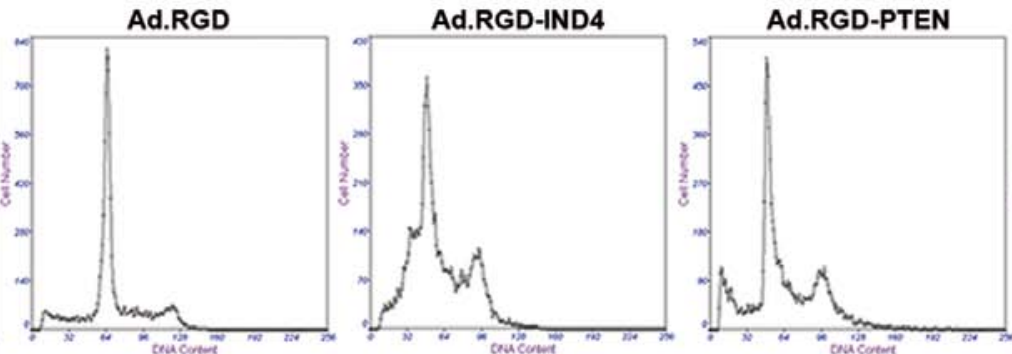

Ad.RGD-ING4-PTEN

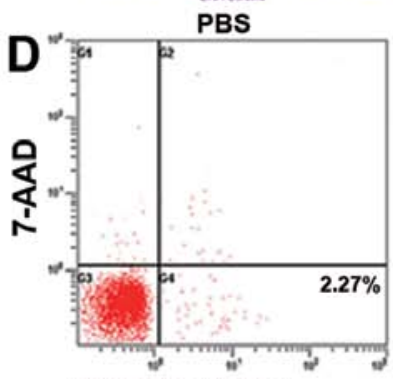

Ad.RGD
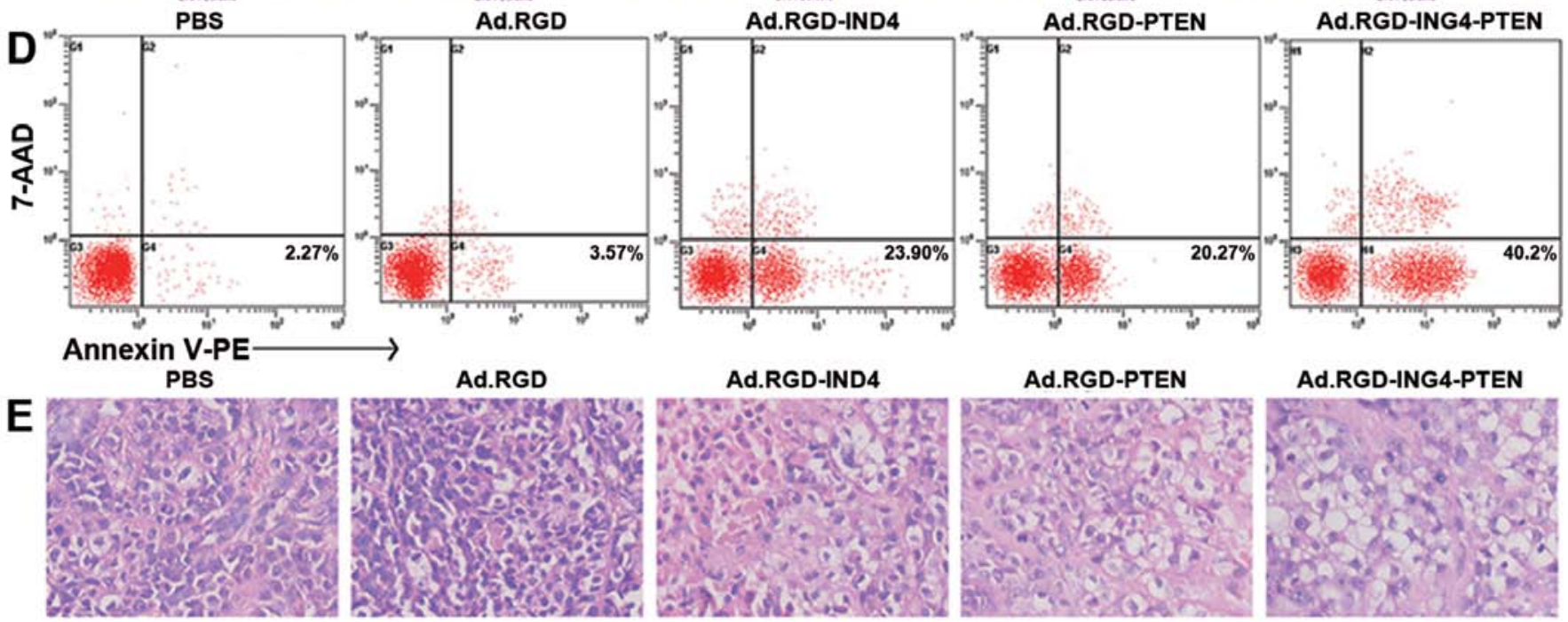

Figure 3. Ad.RGD-ING4-PTEN enhances G2/M phase arrest and apoptosis. (A and B) Cell cycle analysis by flow cytometry in vitro. The CNE human NPC cells were treated for $72 \mathrm{~h}$ with Ad.RGD-ING4-PTEN, Ad.RGD-ING4, Ad.RGD-PTEN, Ad.RGD at the optimal MOI of 50 and PBS. "P<0.05 vs. PBS and Ad.RGD groups; ${ }^{\prime} \mathrm{P}<0.05$ vs. Ad.RGD-ING4 and Ad.RGD-PTEN groups $\left(\mathrm{Q}_{\mathrm{G} 2 / \mathrm{M}}=0.936 ; \mathrm{Q}_{\mathrm{G} 0 / \mathrm{G} 1}=0.861\right)$, one-way repeated measures ANOVA and multiple comparisons, $\mathrm{n}=3$ replicates per condition. (C and D) Flow cytometric analysis of apoptosis in vitro. CNE tumor cells were treated for $72 \mathrm{~h}$ with Ad.RGDING4-PTEN, Ad.RGD-ING4, Ad.RGD-PTEN, Ad.RGD at the optimal MOI of 50 and PBS. The Annexin V single-positive cells in the total cell population represent early apoptotic cells. "P $<0.05$ vs. $\mathrm{PBS}$ and Ad.RGD groups; ${ }^{\prime} \mathrm{P}<0.05$ vs. Ad.RGD-ING4 and Ad.RGD-PTEN groups $(\mathrm{Q}=1.042)$, one-way repeated measures ANOVA and multiple comparisons, $\mathrm{n}=3$ replicates per condition. (E) The morphology of tumor tissues in the five different groups ( $\mathrm{x} 400$ ).

tumors were isolated at 20 days after treatment and the weight (Fig. 2C) was measured. Compared with the Ad.RGD-ING4and Ad.RGD-PTEN-treated groups, the growth of xenografted tumors in nude mice was observably retarded in the Ad.RGDING4-PTEN-treated group $\left(\mathrm{P}<0.05 ; \mathrm{Q}_{\text {volume }}=0.98\right.$ and 1.02 at days 15 and 20 after treatment, respectively, and $\mathrm{Q}_{\text {weight }}=1.07$ ), indicating that Ad.RGD-ING4-PTEN administration significantly suppressed CNE xenografted tumor growth in vivo eclipsing the additive effect.

Alteration in cell cycle distribution and enhanced induction of apoptosis by ING4 and PTEN coexpression. To explore the mechanism by which Ad.RGD-ING4-PTEN causes enhanced tumor suppression in CNE tumor cells, the cell cycle profiles and apoptosis of the CNE tumor cells treated for $72 \mathrm{~h}$ with Ad.RGD-ING4-PTEN, Ad.RGD-ING4, Ad.RGD-PTEN, Ad.RGD (MOI of 50) or PBS were investigated by flow cytometric analysis of PI staining, and Annexin V-PE/7-AAD double staining, respectively. As shown in Fig. 3A and C, compared with the PBS (7.73\%) and Ad.RGD (9.27\%) control groups, a significant increase in the $\mathrm{G} 2 / \mathrm{M}$ phase population was observed in the Ad.RGD-ING4 (24.00\%), Ad.RGD-PTEN $(22.90 \%)$ and Ad.RGD-ING4-PTEN (38.77\%) groups $(\mathrm{P}<0.05)$. In contrast, a significant reduction in the G0/G1 
A
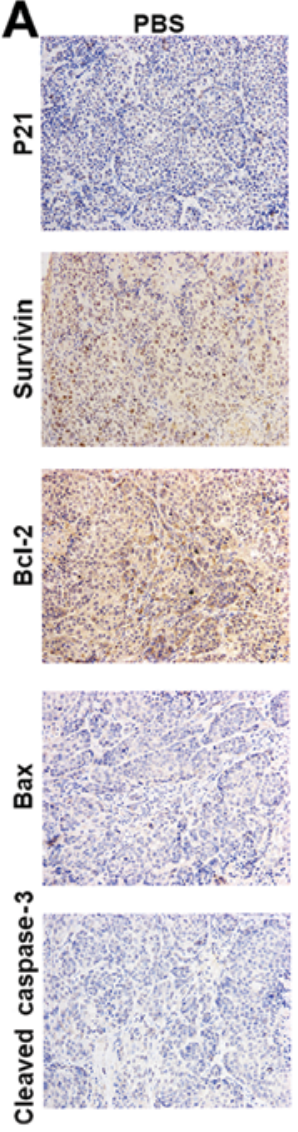

C

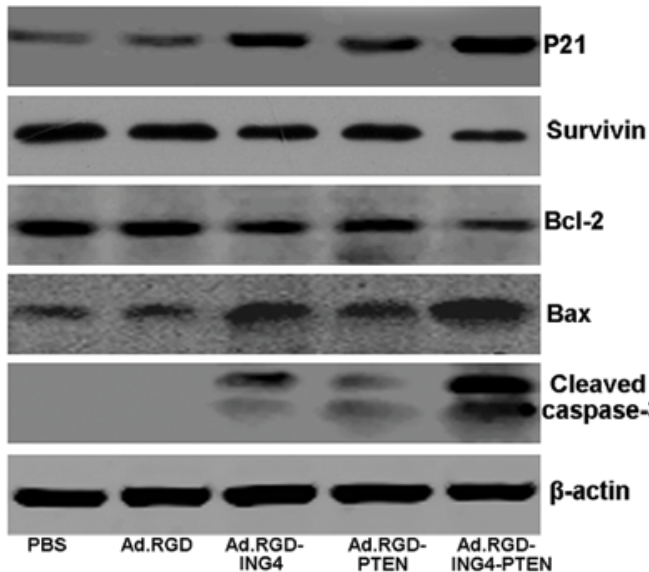

Ad.RGD
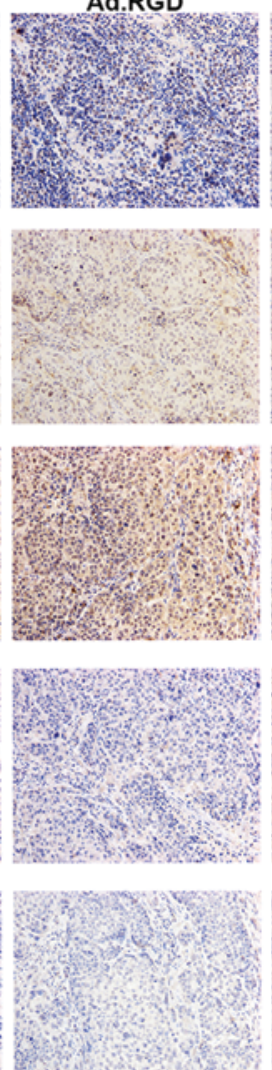
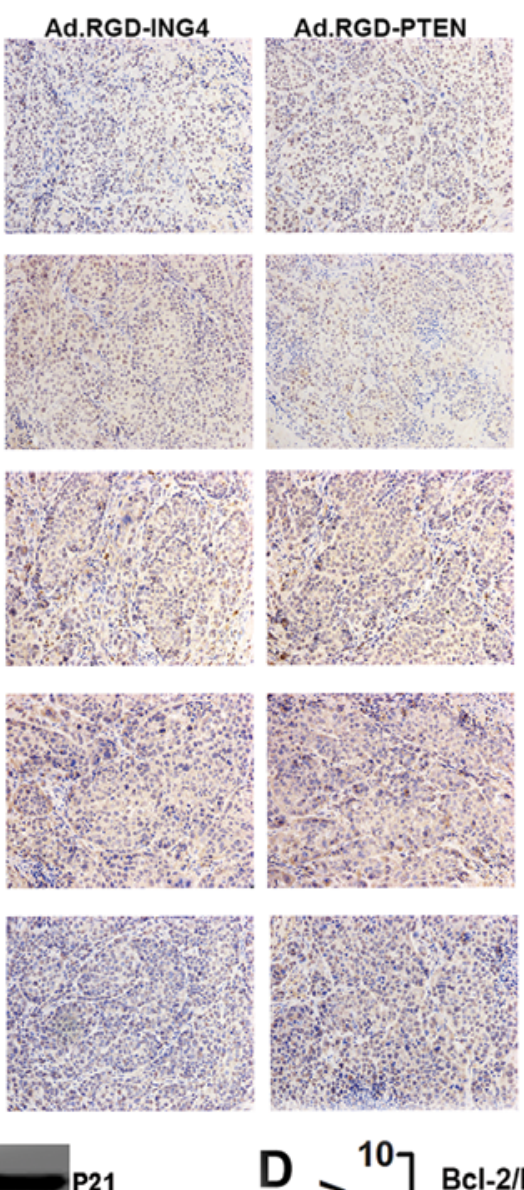

D

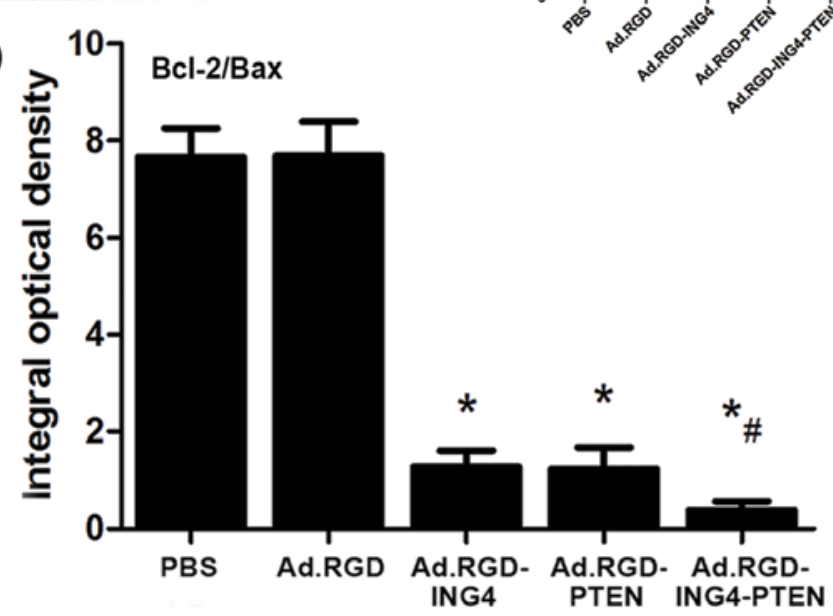

Figure 4. Ad.RGD-ING4-PTEN regulates the intrinsic and extrinsic apoptotic pathways. (C) Western blot analysis of cell cycle- and apoptosis-related proteins. The CNE human nasopharyngeal carcinoma cells were treated for $48 \mathrm{~h}$ with Ad.RGD-ING4-PTEN, Ad.RGD-ING4, Ad.RGD-PTEN or Ad.RGD at the optimal MOI of 50 or PBS. Total cellular lysates were subjected to western blot analysis of P21, survivin, Bcl-2, Bax, caspase-3 and $\beta$-actin expression. Protein expression was normalized to the control $\beta$-actin. (A, B and D) Immunohistochemical analysis of cell cycle- and apoptosis-related proteins. Representative images of immunohistochemical detection of P21, survivin, Bcl-2, Bax, and cleaved caspase-3 in xenografted CNE human nasopharyngeal carcinoma tumors (x200). The immunostaining intensity of P21, survivin, Bcl-2, Bax, and cleaved caspase-3 was quantified as integral optical density (IOD) by Image-Pro Plus 6.0 software. ${ }^{*}<0.05$ vs, PBS and Ad.RGD groups, ${ }^{*} \mathrm{P}<0.05$ vs. Ad.RGD-ING4 and Ad.RGD-PTEN groups $\left(\mathrm{Q}_{\mathrm{P} 21}=0.912, \mathrm{Q}_{\mathrm{Survivin}}=0.928, \mathrm{Q}_{\mathrm{Bc}-2}=1.016\right.$, $\mathrm{Q}_{\mathrm{Bax}}=1.161, \mathrm{Q}_{\text {cleaved caspase-3}}=0.927$ and $\mathrm{Q}_{\mathrm{Bcl}-2 / \mathrm{Bax}}=1.158$ ), one-way repeated measures (ANOVA) and multiple comparisons, $\mathrm{n}=5$ replicates per condition, $\mathrm{n}=5$ observations per representative section. Data shown are representative of three independent experiments.

phase population was observed in the Ad.RGD-ING4 (50.94\%), Ad.RGD-PTEN (55.17\%) and Ad.RGD-ING4-PTEN (40.87\%) groups compared with the PBS (73.57\%) and Ad.RGD $(70.4 \%)$ control groups $(\mathrm{P}<0.05)$. Compared with the single Ad.RGD-ING4- and Ad.RGD-PTEN-treated groups, the $\mathrm{G} 2 / \mathrm{M}$ phase and $\mathrm{G} 0 / \mathrm{G} 1$ phase populations of CNE tumor cells in vitro were significantly increased and decreased, respec- tively, in the Ad.RGD-ING4-PTEN-treated group ( $\mathrm{P}<0.05$; $\left.\mathrm{Q}_{\mathrm{G} 2 / \mathrm{M}}=0.936 ; \mathrm{Q}_{\mathrm{G} 0 / \mathrm{G} 1}=0.861\right)$. In addition, Ad.RGD-ING4PTEN treatment resulted in early apoptosis in $40.20 \%$ of CNE tumor cells, whereas early apoptosis was detected in only 2.27, 3.57, 23.9 and $20.27 \%$ of CNE tumors cells in the PBS, Ad.RGD, Ad.RGD-ING4 and Ad.RGD-PTEN treated groups, respectively. Compared with the single Ad.RGD-ING4- and 


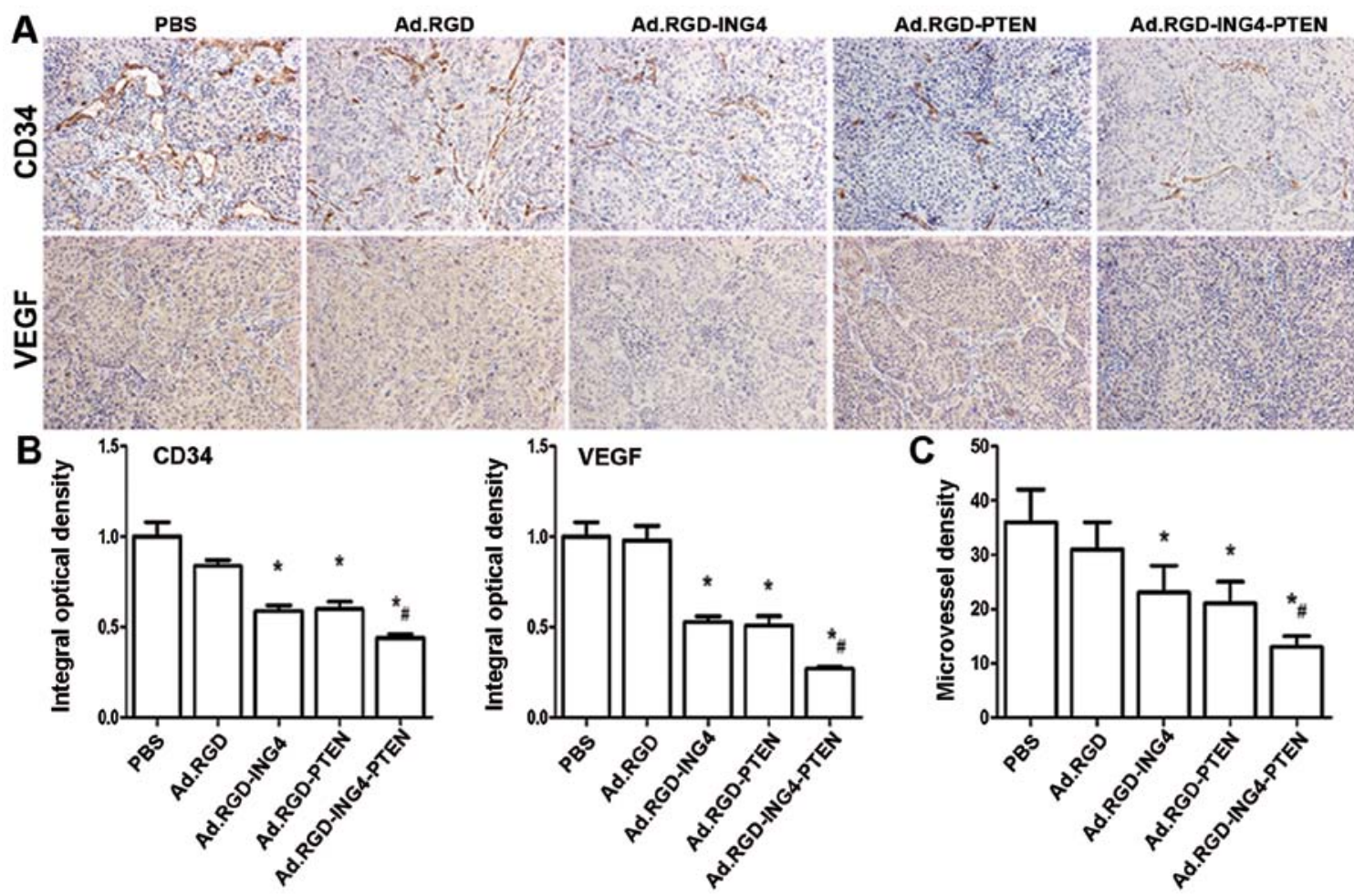

Figure 5. Ad.RGD-ING4-PTEN enhances the reduction in tumor angiogenesis. (A) Representative images of the immunohistochemical detection of CD34 and VEGF in s.c. xenografted CNE human nasopharyngeal carcinoma tumors (x200). (B) The integral optical density of CD34 and VEGF immunostaining intensity quantified by Image-Pro Plus 6.0 software. ${ }^{*} \mathrm{P}<0.05$ vs, $\mathrm{PBS}$ and Ad.RGD groups; ${ }^{*} \mathrm{P}<0.05$ vs. Ad.RGD-ING4 and Ad.RGD-PTEN groups $\left(\mathrm{Q}_{\mathrm{CD} 34}=0.882\right.$, $\mathrm{Q}_{\mathrm{VEGF}}=1.132$ ), one-way repeated measures ANOVA and multiple comparisons, $\mathrm{n}=5$ replicates per condition, $\mathrm{n}=5$ observations per representative section. (C) Tumor MVD estimated by CD34 immunostaining under microscopy (x400). ${ }^{*} \mathrm{P}<0.05$ vs. PBS and Ad.RGD groups; ${ }^{*} \mathrm{P}<0.05$ vs. Ad.RGD-ING4 and Ad.RGD-PTEN groups $\left(\mathrm{Q}_{\mathrm{MVD}}=1.031\right)$, one-way repeated measures ANOVA and multiple comparisons, $\mathrm{n}=5$ replicates per condition, $\mathrm{n}=5$ observations per representative section. Data shown are representative of three independent experiments.

Ad.RGD-PTEN-treated groups, Ad.RGD-ING4-PTEN treatment more efficiently induced apoptosis with an additive effect $(\mathrm{P}<0.05 ; \mathrm{Q}=1.042)$ (Fig. 3B and D).

To further evaluate the induction of apoptosis in vivo, we observed the cellular morphology and karyomorphism of the treated and untreated s.c. xenografted CNE tumors by light microscopy (Fig. 3E). The Ad.RGD-ING4-PTEN group displayed degeneration, and necrosis of tumor cells, as wells as nuclear dissolution. Tumor cell degeneration and necrosis were also observed in the Ad.RGD-ING4 and Ad.RGD-PTEN groups but compared to the Ad.RGD-ING4-PTEN group, the effects were less marked. In the PBS and Ad.RGD groups, tumor cell degeneration and necrosis were not obviously detected.

Ad.RGD-ING4-PTEN cooperatively regulates the intrinsic and extrinsic apoptotic pathways. The potential molecular mechanism responsible for the Ad.RGD-ING4-PTEN-induced increase in antitumor activity was further investigated in western blot and immunohistochemical analyses of the expression of cell cycle- and apoptosis-related proteins such as P21, Bcl-2, Bax, survivin and caspase-3 both in vitro and in vivo. As shown in Fig. 4, the expression of P21 and Bax in the Ad.RGD-ING4, Ad.RGD-PTEN and Ad.RGD-ING4-PTEN groups was obviously increased, whereas Bcl-2 and survivin was decreased. Cleaved caspase-3 was also detected in these groups, but not in the PBS and Ad.RGD groups. Furthermore, Ad.RGD-ING4-PTEN treatment obtained an additive effect on the altered expression of P21, Bcl-2, Bax, survivin and cleaved caspase-3, which are involved in the activation of the intrinsic and extrinsic apoptotic pathways. These observations indicated that Ad.RGD-ING4-PTEN additively suppresses $\mathrm{CNE}$ cell growth and induces apoptosis in a manner that is closely related with the adenovirus-mediated ING4 and PTEN coexpression. This effect is likely to be mediated by cooperative regulation of the intrinsic and extrinsic apoptotic pathways $\left(\mathrm{P}<0.05, \mathrm{Q}_{\mathrm{P} 21}=0.912, \mathrm{Q}_{\text {Survivin }}=0.928, \mathrm{Q}_{\mathrm{Bcl}-2}=1.016, \mathrm{Q}_{\mathrm{Bax}}=1.161\right.$, $\mathrm{Q}_{\text {cleaved caspase-3 }}=0.927$ and $\mathrm{Q}_{\mathrm{Bcl}-2 / \mathrm{Bax}}=1.158$ ).

Enhanced reduction of MVD by Ad.RGD-ING4-PTEN. To examine the combined effect of RGD-modified adenovirusmediated ING4 and PTEN coexpression on tumor angiogenesis in vivo, we evaluated the MVD in s.c. xenografted CNE human NPC tumors on the basis of CD34 immunohistochemical analysis. The CD34-positive expression was mainly manifested as brownish yellow or brownish granules in vascular endothelial cells of CNE human NPC xenografted tumors (Fig. 5A). Compared with the PBS and Ad.RGD control groups, the CD34 expression of vascular endothelial cells in the Ad.RGD-ING4, Ad.RGD-PTEN and Ad.RGDING4-PTEN groups was weaker (Fig. 5A and $\mathrm{B} ; \mathrm{P}<0.05$ ), indicating that Ad.RGD-ING4-PTEN treatment downregulates CD34 expression in s.c. xenografted CNE human NPC tumor vessels. Furthermore, the MVD (Fig. 5B) in the Ad.RGD-ING4, Ad.RGD-PTEN and Ad.RGD-ING4-PTEN groups was obviously less than that in the PBS and Ad.RGD 
groups $(\mathrm{P}<0.05)$. In addition, Ad.RGD-ING4-PTEN exhibited an overlapping effect on downregulation of CD34 and reduction in MVD in the xenografted CNE human NPC tumors $\left(\mathrm{P}<0.05 ; \mathrm{Q}_{\mathrm{CD} 34}=0.882\right.$ and $\left.\mathrm{Q}_{\mathrm{MVD}}=1.031\right)$. These observations closely correlated with the enhanced growth inhibition of Ad-ING4-PTEN-modified CNE NPC xenografted tumors in the athymic nude mouse model.

Ad.RGD-ING4-PTEN suppresses the expression of the proangiogenic factor VEGF. To investigate whether Ad.RGD-ING4-PTEN affects the production of proangiogenic factors, we estimated vascular endothelial growth factor (VEGF) expression in vivo in the s.c. xenografted CNE human NPC tumors by immunohistochemical analysis. As shown in Fig. 5A and C, VEGF expression in the Ad.RGD-ING4, Ad.RGD-PTEN and Ad.RGDING4-PTEN groups was obviously decreased compared with the PBS and Ad.RGD groups. In addition, Ad.RGDING4-PTEN treatment obtained an overlapping effect on the altered expression of VEGF, indicating that Ad.RGDING4-PTEN gene therapy is capable of suppressing the production of VEGF resulting in the inhibition of tumor growth $\left(\mathrm{P}<0.05, \mathrm{Q}_{\mathrm{VEGF}}=1.132\right)$.

\section{Discussion}

Radiotherapy combined with chemotherapy is the standard treatment paradigm for NPC, but long-term survival remains poor because of the high incidence of local recurrences and distant metastasis. Therefore, it is important to identify a novel treatment for NPC. Multigene-based combination therapy shows therapeutic benefit by targeting multiple pathways. Based on current research that has indicated the potential of ING4 and PTEN as tumor suppressors in cancer therapy, we inferred that antitumor activity can be enhanced by the combined expression of ING4 and PTEN. In this study, we constructed an RGD-modified bicistronic ING4/PTEN adenovirus (Ad.RGD-ING4-PTEN) and assessed its therapeutic effect on CNE human NPC cells in vitro and in vivo in a xenografted tumor model established in athymic nude mice. We demonstrated that the infection ability of the RGD-modified adenovirus was greater than that of the unmodified adenovirus and that Ad.RGD-ING4-PTEN mediated enhanced growth inhibition, apoptosis and G2/M phase arrest in CNE human NPC cells in vitro. Moreover, Ad.RGD-ING4-PTEN additively suppressed in vivo CNE human NPC tumor growth and angiogenesis in xenografted nude mice.

P21 was the first discovered cyclin-dependent kinase inhibitor (CKI) gene, and is a member of the inherently disordered protein (IDP) family. Some studies have shown that P21 actively regulates almost all cyclin-CDK complexes, suggesting that P21 plays a role in multiple aspects of the cell cycle and is an important tumor suppressor gene $(29,30)$. A decrease in the $\mathrm{Bcl}-2 / \mathrm{Bax}$ ratio increases mitochondrial membrane permeability, resulting in release of cytochrome $c$ (Cyt-c), which assembles into a large protein complex, the apoptosome, that activates the caspase family of cell death proteins $(31,32)$. Survivin, which is a highly potent apoptosis inhibitor, is absent in most adult tissues, but is selectively upregulated in numerous human tumors. Survivin upregula- tion reduces apoptosis stimulated by both the intrinsic and extrinsic apoptotic pathways, including FAS ligand, overexpression of Bax and caspases-3, -7 and -8 (33). Ultimately, both pathways signal via initiator caspases and converge at the level of the effector caspases (e.g., caspase-3, -6, and -7) to induce cell death through cleavage of essential cellular proteins (34). To investigate the potential mechanism by which Ad.RGDING4-PTEN enhanced antitumor activity, the expression of the cell cycle- and apoptosis-related proteins, P21, Bcl-2, Bax, survivin and cleaved caspase- 3 was investigated in CNE human NPC cells and s.c. xenografted CNE human NPC tumors was estimated by western blot analysis and immunohistochemical staining. Ad.RGD-ING4-PTEN elicited a cooperative and overlapping effect on upregulation of P21, Bax and cleaved caspase-3 and downregulation of Bcl-2 and survivin leading to activation of the extrinsic and intrinsic apoptotic pathways. These observations may explain the enhanced growth inhibition and apoptosis in CNE tumor cells and xenografted tumors induced by Ad.RGD-ING4-PTEN.

Recent studies have demonstrated that the growth and metastasis of malignant tumors is closely related to angiogenesis, which is therefore a potential target in cancer gene therapy. VEGF plays a significant role in controlling the neoplastic angiogenic process. Recent studies have confirmed that VEGF promotes the growth of arterial, venous and lymphatic endothelial cells both in vitro and in vivo and promotes the survival and migration of endothelial cells $(35,36)$. In our study, we demonstrated that Ad.RGD-ING4-PTEN inhibits CD34 and VEGF expression and reduces MVD in s.c. xenografted CNE human NPC tumors. These results suggest that Ad.RGDING4-PTEN inhibits angiogenesis and inhibits NPC tumor growth by downregulating VEGF expression.

In conclusion, Ad.RGD-ING4-PTEN was shown to enhance growth inhibition and apoptosis of CNE human NPC cells and xenografted tumors. This effect was accompanied by an overlapping effect of the individual genes on upregulation of P21, Bax and cleaved caspase-3 expression and downregulation of Bcl-2 and survivin expression. Moreover, Ad.RGD-ING4-PTEN treatment additively downregulated CD34, VEGF and MVD in s.c. xenografted CNE human NPC tumors. The enhanced antitumor activity generated by Ad.RGD-ING4-PTEN was closely associated with activation of the intrinsic and extrinsic apoptotic pathways and additive inhibition of tumor angiogenesis both in vitro and in vivo. On the basis of this evidence, we believe that cancer gene therapy combining two tumor suppressors, such as ING4 and PTEN, represents an effective and novel therapeutic strategy for NPC and other cancers.

\section{Acknowledgements}

This study was supported by grants from the National Natural Science Foundation of China (no. 81001016) and the Medicine Research Foundation of the Board of Health of Suzhou City (no. SYS201014).

\section{References}

1. Chang ET and Adami HO: The enigmatic epidemiology of nasopharyngeal carcinoma. Cancer Epidemiol Biomarkers Prev 15: $1765-1777,2006$ 
2. Guigay J: Advances in nasopharyngeal carcinoma. Curr Opin Oncol 20: 264-269, 2008.

3. Agulnik M and Epstein JB: Nasopharyngeal carcinoma: current management, future directions and dental implications. Oral Oncol 44: 617-627, 2008.

4. Tao Q and Chan AT: Nasopharyngeal carcinoma: molecular pathogenesis and therapeutic developments. Expert Rev Mol Med 9: 1-24, 2007.

5. Ma BB, Hui EP and Chan AT: Systemic approach to improving treatment outcome in nasopharyngeal carcinoma: current and future directions. Cancer Sci 99: 1311-1318, 2008.

6. Schnell O, Krebs B, Wagner E, et al: Expression of integrin alphavbeta3 in gliomas correlates with tumor grade and is not restricted to tumor vasculature. Brain Pathol 18: 378-386, 2008

7. Katayama K, Furuki R, Yokoyama H, et al: Enhanced in vivo gene transfer into the placenta using RGD fiber-mutant adenovirus vector. Biomaterials 32: 4185-4193, 2011.

8. Park J, Singha K, Son S, Kim J, Namgung R, Yun CO and Kim WJ: A review of RGD-functionalized nonviral gene delivery vectors for cancer therapy. Cancer Gene Ther 19 741-748, 2012

9. O'Neill AM, Smith AN, Spangler EA, et al: Resistance of canine lymphoma cells to adenoviral infection due to reduced cell surface RGD binding integrins. Cancer Biol Ther 11: 651-658, 2011.

10. Shiseki M, Nagashima M, Pedeux RM, et al: p29ING4 and p28ING5 bind to p53 and p300, and enhance p53 activity. Cancer Res 63: 2373-2378, 2003.

11. Ythier D, Larrieu D, Brambilla C, Brambilla E and Pedeux R The new tumor suppressor genes ING: genomic structure and status in cancer. Int J Cancer 123: 1483-1490, 2008.

12. Palacios A, Moreno A, Oliveira BL, et al: The dimeric structure and the bivalent recognition of $\mathrm{H} 3 \mathrm{~K} 4 \mathrm{me} 3$ by the tumor suppresso ING4 suggests a mechanism for enhanced targeting of the HBO1 complex to chromatin. J Mol Biol 396: 1117-1127, 2010.

13. Li J, Martinka M and Li G: Role of ING4 in human melanoma cell migration, invasion and patient survival. Carcinogenesis 29 $1373-1379,2008$

14. Garkavtsev I, Kozin SV, Chernova O, et al: The candidate tumour suppressor protein ING4 regulates brain tumour growth and angiogenesis. Nature 428: 328-332, 2004.

15. Liu Y, Yu L, Wang Y, Zhang Y, Wang Y and Zhang G: Expression of tumor suppressor gene ING4 in ovarian carcinoma is correlated with microvessel density. J Cancer Res Clin Oncol 138: 647-655, 2012

16. Zeng ZL, Li FJ, Gao F, Sun DS and Yao L: Upregulation of miR-650 is correlated with down-regulation of ING4 and progression of hepatocellular carcinoma. J Surg Oncol 107: 105-110, 2013

17. Xie Y, Sheng W, Miao J, Xiang J and Yang J: Enhanced antitumor activity by combining an adenovirus harboring ING4 with cisplatin for hepatocarcinoma cells. Cancer Gene Ther 18 176-188, 2011.

18. Zhao Y, Su C, Zhai H, Tian Y, Sheng W, Miao J and Yang J: Synergistic antitumor effect of adenovirus-mediated hING4 gene therapy and (125)I radiation therapy on pancreatic cancer. Cancer Lett 316: 211-218, 2012.
19. Ling C, Xie Y, Zhao D, Zhu Y, Xiang J and Yang J: Enhanced radiosensitivity of non-small-cell lung cancer (NSCLC) by adenovirus-mediated ING4 gene therapy. Cancer Gene Ther 19: 697-706, 2012

20. Unoki M, Shen JC, Zheng ZM and Harris CC: Novel splice variants of ING4 and their possible roles in the regulation of cell growth and motility. J Biol Chem 281: 34677-34686, 2006.

21. Salmena L, Carracedo A and Pandolfi PP: Tenets of PTEN tumor suppression. Cell 133: 403-414, 2008.

22. Leslie NR and Foti M: Non-genomic loss of PTEN function in cancer: not in my genes. Trends Pharmacol Sci 32: 131-140, 2011.

23. Liu P, Cheng H, Roberts TM and Zhao JJ: Targeting the phosphoinositide 3-kinase pathway in cancer. Nat Rev Drug Discov 8: 627-644, 2009.

24. Leslie NR, Yang X, Downes CP and Weijer CJ: PtdIns $(3,4,5)$ $\mathrm{P}(3)$-dependent and -independent roles for PTEN in the control of cell migration. Curr Biol 17: 115-125, 2007.

25. Putz U, Howitt J, Doan A, Goh CP, Low LH, Silke J and Tan SS: The tumor suppressor PTEN is exported in exosomes and has phosphatase activity in recipient cells. Sci Signal 5: ra70, 2012.

26. Hopkins BD, Fine B, Steinbach N, et al: A secreted PTEN phosphatase that enters cells to alter signaling and survival. Science 341: 399-402, 2013.

27. Weidner N: Current pathologic methods for measuring intratumoral microvessel density within breast carcinoma and other solid tumors. Breast Cancer Res Treat 36: 169-180, 1995.

28. Wang W, Qin SK, Chen BA and Chen HY: Experimental study on antitumor effect of arsenic trioxide in combination with cisplatin or doxorubicin on hepatocellular carcinoma. World J Gastroenterol 7: 702-705, 2001.

29. Jung YS, Qian Y and Chen X: Examination of the expanding pathways for the regulation of $\mathrm{p} 21$ expression and activity. Cell Signal 22: 1003-1012, 2010.

30. Blain SW: Switching cyclin D-Cdk4 kinase activity on and off. Cell Cycle 7: 892-898, 2008

31. Zhou C, Li X, Du W, et al: Antitumor effects of ginkgolic acid in human cancer cell occur via cell cycle arrest and decrease the Bcl-2/Bax ratio to induce apoptosis. Chemotherapy 56: 393-402, 2010.

32. Lee JS, Jung WK, Jeong MH, Yoon TR and Kim HK: Sanguinarine induces apoptosis of HT-29 human colon cancer cells via the regulation of $\mathrm{Bax} / \mathrm{Bcl}-2$ ratio and caspase-9-dependent pathway. Int J Toxicol 31: 70-77, 2012.

33. Church DN and Talbot DC: Survivin in solid tumors: rationale for development of inhibitors. Curr Oncol Rep 14: 120-128, 2012.

34. Riedl SJ and Shi Y: Molecular mechanisms of caspase regulation during apoptosis. Nat Rev Mol Cell Biol 5: 897-907, 2004

35. Roskoski R Jr: Vascular endothelial growth factor (VEGF) signaling in tumor progression. Crit Rev Oncol Hematol 62: 179-213, 2007.

36. Canavese $M$ and Spaccapelo R: Protective or pathogenic effects of vascular endothelial growth factor (VEGF) as potential biomarker in cerebral malaria. Pathog Glob Health 108: 67-75, 2014. 